\title{
Activating TSHR Gene Mutation
}

National Cancer Institute

\section{Source}

National Cancer Institute. Activating TSHR Gene Mutation. NCI Thesaurus. Code C132060.

Mutation(s) in the TSHR gene resulting in increased function of the thyroid stimulating hormone receptor. 